\title{
Design of a Switching Controller for Adaptive Disturbance Attenuation with Guaranteed Stability
}

\author{
Giorgio Battistelli ${ }^{1}$, Alireza Karimi ${ }^{2}$, Daniela Selvi ${ }^{1}$, and Alberto Tesi ${ }^{1}$
}

\begin{abstract}
In this paper, a new algorithm is proposed for the design of a family of controllers to be used within an adaptive switching control scheme. The resulting switching controller is able to attenuate the effects of disturbances having uncertain and possibly time-varying characteristics, as well as to ensure stability under arbitrary switching sequences. Specifically, the stability requirement is addressed within the synthesis of the set of controllers by imposing some constraints in LMI form. The overall synthesis algorithm is formulated in terms of convex optimization problems, which can be solved by means of standard tools. The validity of the proposed solution is underlined by showing simulation results on an adaptive optics case study.
\end{abstract}

\section{INTRODUCTION}

The Adaptive Switching Control (ASC) paradigm has emerged as a solution to deal with the unavoidable uncertainty which characterizes the model of the plant [1]-[10], and, more recently, to deal with the problem of the attenuation of disturbances with uncertain and possibly time-varying characteristics [11], [12]. With respect to classical Adaptive Control schemes, ASC enjoys some positive features, such as modularity, that is, the independence between the synthesis step and the adaptation mechanism. On the other hand, it is well known that, even if all the synthesized controllers are stabilizing, their employment in an ASC scheme under arbitrary switching sequences may result in an unstable overall closed-loop system. Classical approaches [13]-[15] usually deal with this problem by verifying the stability of the switching system a-posteriori, then adopting solutions such as imposing a dwell-time (i.e., a minimum time interval between two switching instants), or increasing the hysteresis constant (i.e., the minimum difference between performance functionals related to the controllers that allows the replacement of the controller which is currently in feedback with the plant by a potentially better one). It is clear, however, that such mechanisms can make the ASC system less prompt to reconfigure the control action in response to variations of the operating condition.

This paper addresses the problem of the attenuation of disturbances with uncertain characteristics. It is assumed that the uncertainty is such that a single robust controller cannot provide a satisfactory performance level in all the possible

\footnotetext{
${ }^{1}$ Giorgio Battistelli, Daniela Selvi and Alberto Tesi are with the Dipartimento di Ingegneria dell'Informazione (DINFO), Università di Firenze, Via Santa Marta 3, 50139 Firenze, Italy giorgio.battistelli@unifi.it, daniela.selvi@unifi.it, alberto.tesi@unifi.it

${ }^{2}$ Alireza Karimi is with Laboratoire d'Automatique, Ecole Polytechnique Fédérale de Lausanne (EPFL), CH-1015 Lausanne, Switzerland alireza.karimi@epfl.ch
}

operating conditions. Hence, following [11], an ASC scheme is adopted; a family of stabilizing controllers is synthesized, each one providing a certain attenuation level for a specific operating condition, and a high-level logic selects at each time instant the one providing the best potential performance according to a pre-defined rule. If the switching is guaranteed to stop in finite time, the stability of the overall system can be ensured, provided that all the controllers are stabilizing; however, in general situations (for example, if the uncertain disturbance characteristics are also time-varying), the switching mechanism could be persistent. In the previous work [11], the problem of ensuring the stability of the overall closed-loop system has been addressed by means of a specific architecture of the multi-controller involving a switching mechanism between the Youla parameters related to the controllers. However, the complexity of such an architecture is highly influenced by the plant order.

In this paper, we propose a different technique for dealing with the problem of stability under arbitrary switching, by formulating this requirement as a constraint to be satisfied directly within the synthesis of the set of controllers. In particular, a sufficient condition for the stability of the closed-loop switching system is provided by means of LMI constraints. The proposed method allows one to switch directly between the controllers, making the multi-controller architecture independent of the plant order. As an additional contribution, we propose an algorithm for the design of a family of linear time invariant controllers able to guarantee stability of the closed-loop system under arbitrary switching as well as a satisfactory disturbance attenuation in any possible operating condition. Specifically, we consider the synthesis method proposed in [16] and extend it to the design of a switching controller with guaranteed stability. With this respect, notice that in [17] the approach of [16] has already been applied to active disturbance attenuation within an adaptive gain-scheduling scheme, but stability for fast variations of the disturbance characteristics in that context cannot be guaranteed. The proposed design technique turns out to be well suited to dealing with the context of disturbances described by uncertain models (for example, when only empirical estimates of the disturbance spectra are available) for the synthesis of a set of controllers for an ASC architecture. In fact, this design approach, combined with the stability constraints, allows one to formulate the overall synthesis algorithm in terms of convex optimization problems, which can be solved by means of standard tools. 


\section{PROBLEM FORMULATION}

The problem of interest is that of controlling a single-input single-output (SISO) linear time-invariant (LTI) dynamical system whose input-output behavior can be described as

$$
\mathcal{P}: y(t)=P(d) u(t)+\nu(t),
$$

with the plant $\mathcal{P}$ described by the transfer function $P(d)$, where $d$ is the unit backward shift operator. Discrete time instants are denoted by $t \in \mathbb{Z}_{+}, \mathbb{Z}_{+}:=\{0,1, \cdots\} ; y(t)$ is the output of the system, and $u(t)$ is the control input; finally, $\nu(t)$ is a bounded disturbance with uncertain and possibly time-varying characteristics.

The aim is that of regulating $y(t)$ around zero by attenuating the disturbance $\nu(t)$. In this paper, we assume that the action provided by a single robust controller is not able to provide a satisfactory performance level for all the possible operating conditions, and we propose a solution based on the ASC paradigm; a high-level switching logic supervises a family of pre-designed controllers by selecting, at each time instant, the one providing the best potential performance according to a pre-defined rule. The controllers are all stabilizing, and, for any possible operating condition, there exists at least one controller able to achieve a certain performance level. Let $\mathscr{C}:=\left\{\mathcal{C}_{i}, i \in \overleftarrow{N}\right\}$ be the family of pre-designed candidate controllers, where $\overleftarrow{N}:=\{1,2, \cdots, N\}$. The $i$ th controller has transfer function $C_{i}(d)$. We denote by $\sigma(\cdot): \mathbb{Z}_{+} \rightarrow \overleftarrow{N}$ the switching signal identifying which controller in $\mathscr{C}$ is selected by the supervisor at each time instant to be in feedback with the plant. Taking into account this notation, the switching controller is denoted by $\mathcal{C}_{\sigma(t)}$, meaning that, on all the time intervals on which $\sigma(t)$ is constant and equal to a certain $i$, the multi-controller takes the form of a LTI system having transfer function equal to $C_{i}(d)$. We refer to $\left(\mathcal{P} / \mathcal{C}_{\sigma(t)}\right)$ as the closed-loop system involving the feedback interconnection of $\mathcal{C}_{\sigma(t)}$ with $\mathcal{P}$.

In order to infer the potential behavior of the candidate controllers belonging to $\mathscr{C}$, the supervisory unit compares, at each time instant, the values assumed by test functionals $\Pi_{i}(t), i \in \overleftarrow{N}$, each one related to a controller $\mathcal{C}_{i} \in \mathscr{C}$. This mechanism allows one to evaluate the potential performance of each controller $\mathcal{C}_{i}$ without the need of any experiment involving the feedback of the plant with $\mathcal{C}_{i}$.

The test functionals are supposed to be bounded and computable in real time on the basis of the plant input/output data; thanks to the modularity of the ASC paradigm, the choice of the test functionals is immaterial for the subsequent developments. Details about possible definitions of $\Pi_{i}(t)$ can be found for example in [11], [12].

A common choice for the switching rule, according to which the supervisor selects the (potentially) best controller, is to adopt the Hysteresis Switching Logic [1]

$$
\sigma(t+1)=\arg \min _{i \in \overleftarrow{N}}\left\{\Pi_{i}(t)-h \delta_{i \sigma(t)}\right\},
$$

where $\delta_{i j}$ is the Kronecker delta and $h>0$ is the hysteresis constant. The switching mechanism is started at a time instant $t_{0} \geq 0$, and, for any $t_{\leq} \leq t_{0}, \sigma(t)$ is kept constant and equal to an arbitrary $i_{0} \in \overleftarrow{N}$.

It is important to notice that, when the switching is persistent, the stability of $\left(\mathcal{P} / \mathcal{C}_{\sigma(t)}\right)$ is not ensured even if all the controllers $\mathcal{C}_{i}, i \in \overleftarrow{N}$, are stabilizing. Specifically, a persistent switching is likely to happen when the disturbance characteristics keep varying with time. Thus, one of the main problems that any ASC technique has to deal with is that of guaranteeing the stability of the closed-loop switching system under arbitrary switching. This issue has been addressed in the context of adaptive disturbance attenuation in [11], where a solution is proposed which relies on a specific architecture of the multi-controller based on a switching mechanism between the Youla parameters associated to the stabilizing controllers in $\mathscr{C}$; the complexity of the adopted architecture, however, is unavoidably influenced by the plant order. In the present work, on the other hand, we propose to address the stability problem directly within the design of the controller set; an algorithm is provided for a convexoptimization-based design of a switching control system able to ensure the stability of the closed-loop system under arbitrary switching as well as to attenuate disturbances with uncertain and possibly time-varying characteristics.

\section{STABILITY UNDER ARBITRARY SWITCHING}

This section is focused on the problem of stability of the overall closed-loop system under arbitrary switching. The solution that we propose relies on sufficient conditions which can be expressed in terms of LMI constraints, and thus can be easily integrated in a number of performance-oriented design techniques. Further, the proposed approach allows one to adopt a simple multi-controller architecture by switching directly between the controllers in $\mathscr{C}$, making the multicontroller structure independent of the plant model.

The idea is based on the well known fact that a sufficient condition ensuring stability of a switching system is the existence of a common quadratic Lyapunov function between the subsystems corresponding to the different possible configurations.

Before stating the main result, it is useful to recall the following definition from [18].

Definition 1: Two matrices $F$ and $V$ in $\mathbb{R}^{m \times m}$, with $m$ a positive integer, are called a strictly positive real pair (SPRpair) if the transfer matrix $H(d)$ defined by the quadruple $(F, I, F-V, I)$, i.e., $H(d)=d(F-V)(I-d F)^{-1}+I$, is SPR.

Then the following proposition can be stated [18], in which the symbol $\star$ is introduced to denote a symmetric block.

Proposition 1: Given $F$ and $V$ in $\mathbb{R}^{m \times m}$, if they are a SPR-pair, then they are both stable with a common Lyapunov matrix $Q>0$ and the following inequalities are equivalent:

$$
\begin{gathered}
{\left[\begin{array}{cc}
F^{\top} Q F-Q & \star \\
Q F-F+V & Q-2 I
\end{array}\right]<0} \\
{\left[\begin{array}{cc}
V^{\top} Q V-Q & \star \\
Q V-V+F & Q-2 I
\end{array}\right]<0 .}
\end{gathered}
$$


Let $\left(A_{C_{i}}, B_{C_{i}}, C_{C_{i}}, D_{C_{i}}\right)$ be stabilizable and detectable realizations of $C_{i}(d)$ having the same order for any $i \in \overleftarrow{N}$ (for example, when all the $C_{i}(d)$ have the same order, controllable/observable canonical forms can be used). Further, let $\left(A_{P}, B_{P}, C_{P}\right)$ be a minimal realization of $P(d)$. Then the $i$-th closed-loop state matrix is defined as

$$
A_{C L, i}=\left[\begin{array}{cc}
A_{P}-B_{P} D_{C_{i}} C_{P} & B_{P} C_{C_{i}} \\
-B_{C_{i}} C_{P} & A_{C_{i}}
\end{array}\right] .
$$

With respect to the multi-controller, let the shared-state architecture

$$
\mathcal{C}_{\sigma(t)}:\left\{\begin{array}{l}
x_{C}(t+1)=A_{C_{\sigma(t)}} x_{C}(t)-B_{C_{\sigma(t)}} y(t) \\
u(t)=C_{C_{\sigma(t)}} x_{C}(t)-D_{C_{\sigma(t)}} y(t)
\end{array}\right.
$$

be adopted, with $x_{C}$ the controller state.

The following result can now be presented (the proof is omitted due to space limitations; useful guidelines can be found in [18]).

Theorem 1: Let $A_{C L, i}, i \in \overleftarrow{N}$, be the closed-loop state matrices, defined as in (5). Further, let $M_{i}, i \in \overleftarrow{N}$, be stable matrices, and $T$ a non-singular matrix, and assume that $M_{i}$ and $T^{-1} A_{C L, i} T$ are SPR-pair, $\forall i \in \overleftarrow{N}$. Finally, let the multicontroller be implemented as in (6). Then, if there exists a symmetric positive-definite matrix $G$ such that

$$
\left[\begin{array}{cc}
M_{i}^{\top} G M_{i}-G & \star \\
G M_{i}-M_{i}+T^{-1} A_{C L, i} T & G-2 I
\end{array}\right]<0, \quad \forall i \in \overleftarrow{N}
$$

the overall closed-loop switching system is exponentially stable under arbitrary switching.

Remark 1: It it is worth underlining that the matrix $T$ must be the same for all the controllers in $\mathscr{C}$ in order to have all the $A_{C L, i}, i \in \overleftarrow{N}$, share the common Lyapunov matrix $T^{-\top} G T^{-1}$ (where $T^{-\top}:=\left(T^{\top}\right)^{-1}$ ). This follows readily by applying the equivalence between (3) and (4) to (7).

In this paper, we consider a set of linearly-parametrized controllers with transfer functions

$$
C_{i}(d)=C\left(d, \rho_{i}\right)=\phi_{i}^{\top}(d) \rho_{i}, \quad \forall i \in \overleftarrow{N}
$$

where $\rho_{i}:=\left[\rho_{i, 1}, \rho_{, 2}, \cdots, \rho_{i, n}\right]^{\top}$ is a $n$-dimensional parameter vector, with $n$ a positive integer, and $\phi_{i}(d):=$ $\left[\phi_{i, 1}, \phi_{i, 2}, \cdots, \phi_{i, n}\right]^{\top}$ is a $n$-dimensional array of stable transfer functions with bounded infinity norm. The choice of the functions in $\phi_{i}(d)$ highly affects the resulting control action and depends on the specific application; some general guidelines can be found in [19].

Thanks to the controller parameterization (8), if, for example, we realize each $C_{i}(d)$ in controllable canonical form, then the matrices $C_{C_{i}}=C_{C}\left(\rho_{i}\right)$ and $D_{C_{i}}=D_{C}\left(\rho_{i}\right)$ are affine functions of the parameter vector $\rho_{i}$, while the matrices $A_{C_{i}}$ and $B_{C_{i}}$ are constant. This in turn implies that each matrix $A_{C L, i}=A_{C L}\left(\rho_{i}\right)$ depends affinely on $\rho_{i}$; thus each inequality (7) becomes an LMI with respect to the unknowns $G$ and $\rho_{i}$.

\section{A. Choice of $M_{i}$ and $T$}

With respect to the choice of the matrices $M_{i}$ and $T$, following [18] we consider a set of $N$ initial fixed-order stabilizing controllers, which can be synthesized according to any design technique (a possible synthesis approach that can be used is described in Section IV). Specifically, let $\bar{A}_{C L, i}$ be the closed-loop state matrix related to the $i$-th controller. Then, the matrices $M_{i}$ and $T$ can be determined by solving the following feasibility problem

$$
\begin{gathered}
{\left[\begin{array}{cc}
\bar{A}_{C L, i}^{\top} G_{i, T} \bar{A}_{C L, i}-G_{i, T} & \star \\
G_{i, T} \bar{A}_{C L, i}-X \bar{A}_{C L, i}+M_{i, T} & G_{i, T}-2 X
\end{array}\right]<0} \\
G_{i, T}>0, \quad \forall i \in \overleftarrow{N}
\end{gathered}
$$

with respect to the unknowns $G_{i, T}, X, M_{i, T}$, where $M_{i, T}:=$ $T^{-\top} M_{i} T^{-1}, G_{i, T}:=T^{-\top} G_{i} T^{-1}, X:=T^{-\top} T^{-1}$. Thus

$$
\begin{aligned}
T & =(\operatorname{chol}(X))^{-1} \\
M_{i} & =T^{\top} M_{i, T} T,
\end{aligned}
$$

where chol denotes the Cholesky factorization. The rationale behind the proposed choice is that, by solving the feasibility problem (9), we aim at finding matrices $M_{i}$ and $T$ such that $M_{i}$ and $T^{-1} \bar{A}_{C L, i} T, \forall i \in \overleftarrow{N}$, are a SPR-pair, i.e., the SPRpair property involving $M_{i}$ and $T$ holds with respect to the initial controllers.

\section{PERFORMANCE-ORIENTED DESIGN}

In this section we will discuss a design technique aiming at providing fixed-order controllers able to attenuate the effects of the disturbance $\nu(t)$ on the output $y(t)$. This approach was originally proposed in [16] for the synthesis of a single robust fixed-order controller for a SISO plant represented by nonparametric models, and has been exploited in [17] in the context of the attenuation of uncertain and time-varying narrow band disturbances by means of a gain-scheduling scheme, which however cannot guarantee the stability for fast variations of the disturbance characteristics. Even if the requirement accounting for stability under arbitrary switching, discussed in Section III, could be in principle integrated in any design technique, in this paper we exploit the approach in [16] for the synthesis of a set of controllers for an ASC architecture, since this method is well suited to dealing with the context of disturbances described by uncertain models (for example, when only empirical estimates of the disturbance spectra are available); further, it addresses the performance problem by means of convex optimization.

The performance criterion, for each $i \in \overleftarrow{N}$, can be defined as

$$
\left\|W_{1, i} S_{i}\right\|_{\infty}<1
$$

where $S_{i}(d)$ is the sensitivity transfer function related to the controller $\mathcal{C}_{i}$, and $W_{1, i}(d)$ is the transfer function of a performance weighting filter for $\mathcal{S}_{i}(d)$.

Remark 2: The choice of the weights $W_{1, i}(d), i \in \overleftarrow{N}$, can be related to the a-priori available information on the disturbance characteristics (see for example [17]). For example, let 
the model related to the disturbance $\nu$ depend on an uncertain parameter vector $\theta$, with $\theta$ belonging to an uncertainty set $\Theta$. We assume that $\Theta$ is such that the task of providing a good level of attenuation of the disturbance cannot be managed by a single robust controller. A possible solution is then to divide the set $\Theta$ into $N$ subsets $\Theta_{1}, \Theta_{2}, \cdots, \Theta_{N}$ and to address the performance objective for each $\mathcal{C}_{i}$ with respect to a subregion $\Theta_{i}$. If we denote by $\psi(\omega, \theta)$ the parameter-dependent power spectral density of the disturbance $\nu$, then each $W_{1, i}(d)$ could be chosen for example such that

$$
\psi(\omega, \theta) \leq\left|W_{1, i}\left(e^{-j \omega}\right)\right|^{2}, \quad \forall \theta \in \Theta_{i} .
$$

Another possibility is that of addressing the performance problem expressed by (12) only in the correspondence of a grid of $N$ values $\theta_{1}, \theta_{2}, \cdots, \theta_{N}$ of $\theta$ in $\Theta$. In this case, we could define $W_{1, i}(d)$ such that $W_{i, 1}\left(e^{-j \omega}\right)$ is a spectral factor of $\psi\left(\omega, \theta_{i}\right)$. Further, if a data series of the disturbance is available for $N$ operating conditions, then the weights could be modeled such that each $W_{i, 1}\left(e^{-j \omega}\right)$ matches, as closely as possible, the frequency profile which can be extracted from the data.

The method described in [16] relies on the idea of linearizing the performance criterion (12) around a desired openloop transfer function $L_{d, i}(d)$ for each $i \in \overleftarrow{N}$. In practice, each $L_{d, i}(d)$ reflects the open-loop behavior corresponding to a desired controller $C_{d, i}(d)$, which the proposed controller design method tries to approximate. The choice of the $L_{d, i}(d)$ will be briefly discussed in Remark 3 .

When the controller parameterization (8) is adopted, each open-loop transfer function $L_{i}(d)$ is linearly-dependent on the parameter vector $\rho_{i}$ as well, since

$$
L_{i}(d)=L\left(d, \rho_{i}\right)=P(d) C\left(d, \rho_{i}\right)=P(d) \phi_{i}^{\top}(d) \rho_{i} .
$$

This means that each point on the Nyquist diagram of $L\left(d, \rho_{i}\right)$ is a linear function of the parameter vector $\rho_{i}$. Hence, as shown in [16], [17], it is possible to obtain the following linear approximation of (12) in the frequencydomain

$$
\begin{gathered}
\left|W_{1, i}\left(e^{-j \omega}\right)\left[1+L_{d, i}\left(e^{-j \omega}\right)\right]\right|-R e\left\{\left[1+L_{d, i}^{*}\left(e^{-j \omega}\right)\right]\right. \\
\left.\times\left[1+P\left(e^{-j \omega}\right) \phi_{i}^{\top}\left(e^{-j \omega}\right) \rho_{i}\right]\right\}<0 \quad \forall \omega
\end{gathered}
$$

where we denote by $L_{d, i}^{*}\left(e^{-j \omega}\right)$ the complex conjugate of $L_{d, i}\left(e^{-j \omega}\right)$, for each $\omega$ and $i \in \overleftarrow{N}$, and by $\operatorname{Re}\{x\}$ the real part of the complex number $x$. In fact, by recalling that $\operatorname{Re}\{x\} \leq|x|$, where $|x|$ is the modulus of $x$, inequality (15) leads to

$$
\begin{aligned}
& \left|W_{1, i}\left(e^{-j \omega}\right)\left[1+L_{d, i}\left(e^{-j \omega}\right)\right]\right|-\left|\left[1+L_{d, i}^{*}\left(e^{-j \omega}\right)\right]\right| \\
& \times\left|\left[1+P\left(e^{-j \omega}\right) \phi_{i}^{\top}\left(e^{-j \omega}\right) \rho_{i}\right]\right|<0 \forall \omega
\end{aligned}
$$

and thus to

$$
\left|W_{1, i}\left(e^{-j \omega}\right)\right|<\left|\left[1+P\left(e^{-j \omega}\right) \phi_{i}^{\top}\left(e^{-j \omega}\right) \rho_{i}\right]\right| \forall \omega,
$$

from which (12) follows.

Further, we underline that the performance criterion expressed by (12) can be improved by taking into account an upper bound $\gamma$ on the $H_{\infty}$ norm, thus leading to the following optimization problem to be solved:

$$
\min _{\gamma, \rho_{i}} \gamma
$$

s.t.

$$
\begin{gathered}
\left|\frac{1}{\gamma} W_{1, i}\left(e^{-j \omega}\right)\left[1+L_{d, i}\left(e^{-j \omega}\right)\right]\right|-R e\left\{\left[1+L_{d, i}^{*}\left(e^{-j \omega}\right)\right]\right. \\
\left.\times\left[1+P\left(e^{-j \omega}\right) \phi_{i}^{\top}\left(e^{-j \omega}\right) \rho_{i}\right]\right\}<0 \forall \omega
\end{gathered}
$$

By adopting the bisection algorithm, thus fixing the value of $\gamma$ at each step, the problem in (18)-(19) is a linear programming problem.

A practical solution for dealing with the problem of the infinite number of constraints in (19) is to impose such constraints only in the correspondence of a finite number of frequencies $\left\{\omega_{1}, \omega_{2}, \cdots, \omega_{K}\right\}$; clearly the choice of $K$ should be such that both the Nyquist diagram of $L\left(e^{-j \omega_{k}}, \rho_{i}\right), k \in$ $\{1,2, \cdots, K\}$, is a sufficiently accurate approximation of the Nyquist diagram of $L\left(e^{-j \omega}, \rho_{i}\right)$, and the problem has a manageable computational burden. Depending on the specific context of application, the $K$ frequencies can be selected by means of either a random or a deterministic approach [16].

\section{A. The proposed design algorithm}

To conclude Section IV, we provide an outline of the algorithm that we propose to design fixed-order controllers providing attenuation capability in the presence of a disturbance with unknown and possibly time-varying characteristics, and ensuring the stability of the overall closed-loop switching system.

\section{Algorithm 1}

Step 0 - Initialization. For each $i \in \overleftarrow{N}$, define: the desired open-loop transfer function $L_{d, i}\left(e^{-j \omega}\right)$; the weight $W_{1, i}\left(e^{-j \omega}\right)$ for the sensitivity function; the length of the parameter vector $\rho_{i}$; the array of basis functions $\phi_{i}$. Define the set $\left\{\omega_{1}, \omega_{2}, \cdots, \omega_{K}\right\}$.

Step 1. Compute $N$ initial stabilizing controllers accounting for the performance requirement only (for example, by solving the optimization problem (18) - (19)).

Step 2. Compute stabilizable and detectable realizations $\left(\bar{A}_{C_{i}}, \bar{B}_{C_{i}}, \bar{C}_{C_{i}}, \bar{D}_{C_{i}}\right)$ having the same order for all the initial controllers (for example, in controllable canonical form); compute a minimal realization of the plant $\left(A_{P}, B_{P}, C_{P}\right)$.

Step 3. Compute the state matrix of the closed-loop system on the basis of the state-space realizations of the plant and of the $i$-th initial controller for all $i \in \overleftarrow{N}$ :

$$
\bar{A}_{C L, i}=\left[\begin{array}{cc}
A_{P}-B_{P} \bar{D}_{C_{i}} C_{P} & B_{P} \bar{C}_{C_{i}} \\
-\bar{B}_{C_{i}} C_{P} & \bar{A}_{C_{i}}
\end{array}\right] .
$$

Step 4. Solve the feasibility problem

$$
\begin{gathered}
{\left[\begin{array}{c}
\bar{A}_{C L, i}^{\top} G_{i, T} \bar{A}_{C L, i}-G_{i, T} \\
G_{i, T} \bar{A}_{C L, i}-X \bar{A}_{C L, i}+M_{i, T} \quad G_{i, T}-2 X
\end{array}\right]<0} \\
G_{i, T}>0, \quad \forall i \in \overleftarrow{N}
\end{gathered}
$$


with respect to the unknowns $M_{i, T}, G_{i, T}, X$ (defined as in Section III-A), and set

$$
\begin{aligned}
T & =(\operatorname{chol}(X))^{-1} \\
M_{i} & =T^{\top} M_{i, T} T .
\end{aligned}
$$

Step 5. Consider a set of controllers having transfer functions $C\left(d, \rho_{i}\right), i \in \overleftarrow{N}$, depending on a parameter vector $\rho_{i}$, and compute stabilizable and detectable state-space realizations $\left(A_{C_{i}}, B_{C_{i}}, C_{C_{i}}, D_{C_{i}}\right)$ having the same order for all of them (for example, in controllable canonical form); compute the corresponding closed-loop state matrices

$$
A_{C L}\left(\rho_{i}\right)=\left[\begin{array}{cc}
A_{P}-B_{P} D_{C_{i}} C_{P} & B_{P} C_{C_{i}} \\
-B_{C_{i}} C_{P} & A_{C_{i}}
\end{array}\right] .
$$

Step 6. Solve the optimization problem

$$
\min _{\gamma, \rho_{i}, G} \gamma
$$

s.t.

$$
\begin{gathered}
\left|\frac{1}{\gamma} W_{1, i}\left(e^{-j \omega}\right)\left[1+L_{d, i}\left(e^{-j \omega}\right)\right]\right|-\operatorname{Re}\left\{\left[1+L_{d, i}^{*}\left(e^{-j \omega}\right)\right]\right. \\
\left.\times\left[1+P\left(e^{-j \omega}\right) \phi^{\top}\left(e^{-j \omega}\right) \rho_{i}\right]\right\}<0 \\
\forall \omega \in\left\{\omega_{1}, \omega_{2}, \cdots, \omega_{K}\right\}, \quad \forall i \in \overleftarrow{N} \\
M_{i}^{\top} G M_{i}-G \quad \quad \quad \star \\
{\left[\begin{array}{cc}
G M_{i}-M_{i}+T^{-1} A_{C L}\left(\rho_{i}\right) T \quad G-2 I
\end{array}\right]<0} \\
\forall i \in \overleftarrow{N}, \quad G>0
\end{gathered}
$$

Step 7. Compute the set of controllers on the basis of the vectors $\hat{\rho}_{i}$ as $C_{i}(d)=\phi^{\top}(d) \hat{\rho}_{i}$, where $\hat{\rho}_{i}$ is solution to the problem (25)-(27).

Remark 3: The performance of the controllers designed by means of the proposed algorithm can be improved by iterating the whole procedure described in Algorithm 1. In practice, the desired open-loop transfer functions $L_{d, i}(d)$ can be re-computed after Step 7 as $L_{d, i}(d)=P(d) \phi_{i}(d)^{\top} \hat{\rho}_{i}$, and then the algorithm can be restarted from Step 1. This iterative version of the algorithm allows one, for example, to initialize all the $L_{d, i}(d)$ equal to a certain $L_{d}(d)$ (thus increasing the probability that a solution to the problem in (25) - (27) exists), and then to approximate open-loop desired behaviors which become, iteratively, more specific for each $i \in \overleftarrow{N}$

\section{AN EXAMPLE}

To illustrate the validity of the proposed design algorithm, in this section we propose simulation results obtained on an adaptive optics system for ground-based telescopes. The aim is that of reducing the image aberration caused by atmospheric turbulence and structural vibrations (arising in several situations, for example in the presence of wind) by means of a deformable mirror, whose shape has to be made, as close as possible, opposite to the wavefront distortion (see Fig. 1). It is worth underlining that to obtain a good approximation of the disturbance (in particular, the turbulence) profile, a high-order model is required; further,

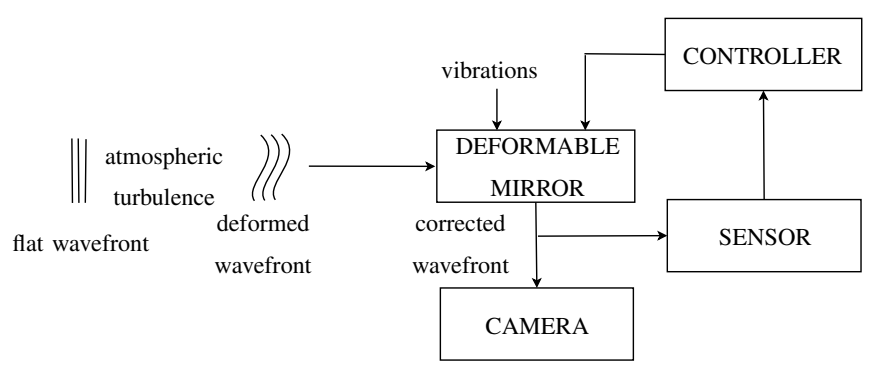

Fig. 1. Principle of functioning of an adaptive optics scheme.

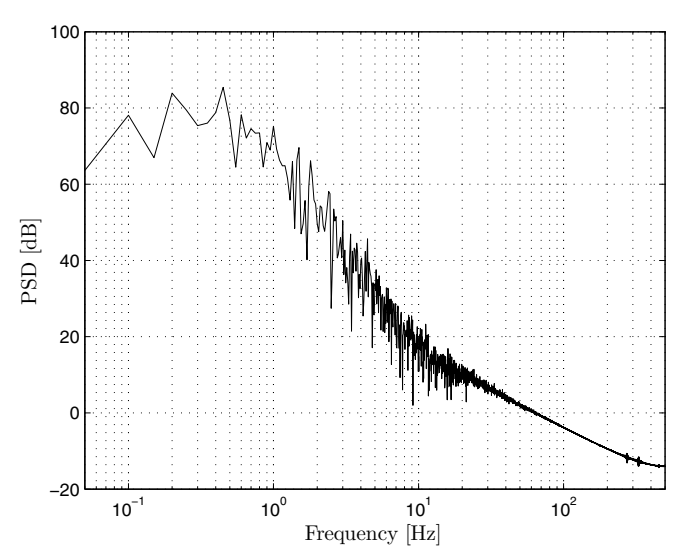

Fig. 2. Turbulence power spectral density related to the optical deformation considered for the tests.

the disturbance profile can vary depending on the operating conditions of the telescope.

We refer to a setting similar to the one described in [20], [21], wherein, thanks to the adoption of a modal basis to express the wavefront distortion, the control architecture is decomposed into a parallel of several SISO loops. In particular, we consider the SISO control loop pertaining to a rigid deformation mode (tip/tilt mode). The interested reader is referred to [20], [21] for further details. Specifically, with respect to the model in (1), the output $y$, to be regulated around zero, is the residual phase after the correction performed by the deformable mirror; the signal $\nu$ is a filtered version of the disturbance resulting from the effects of both turbulence and vibrations on a specific optical deformation.

We assume that the frequency profile of the turbulence does not undergo significant changes in the considered operating conditions; in the tests that follow, we consider for the turbulence a signal having its power spectral density as shown in Fig. 2. We further consider as vibration a damped oscillation having its power spectral density varying with time in the range $\Theta=[10,35] \mathrm{Hz}$.

The design of the control system is performed by following the non-iterative version of Algorithm 1. We synthesize three controllers with the same order equal to 5 ; the size $n$ of the parameter vectors $\rho_{i}$ is set to 6 . The desired open-loop transfer functions $L_{d, i}(d), i=\{1,2,3\}$, are chosen such that the corresponding controllers $C_{d, i}(d)$ exhibit the desired 

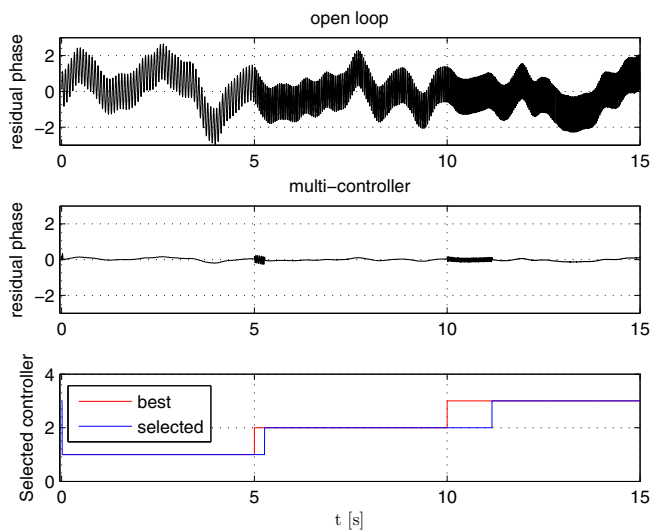

Fig. 3. Response of the system in the presence of turbulence and timevarying oscillations with peak frequency assuming values as in (29): openloop (up) and multi-controller (down).

attenuation capability with respect to both the turbulence and a damped oscillation having its power spectral density in a subset $\Theta_{i} \in \Theta$. Each array $\phi_{i}(d)$ is defined as

$$
\frac{1}{D_{\phi, i}}\left[1, d, d^{2}, \cdots, d^{n-1}\right]^{\top},
$$

where each of the different $D_{\phi, i}, i \in \overleftarrow{N}$, is chosen as the denominator of the controller $C_{d, i}(d)$. The transfer function $W_{1, i}(d)$ is defined taking into account the contribution to the overall disturbance power spectral density provided by both the turbulence and the considered damped oscillation.

We adopt the switching logic described in Section II with test functionals defined in [11], [12], and we let the power spectral density of the damped oscillation have its peak frequency $f$ vary with time as

$$
f:=\left\{\begin{array}{ll}
13 \mathrm{~Hz}, & t \in[0,5) \mathrm{s} \\
22 \mathrm{~Hz}, & t \in[5,10) \mathrm{s} . \\
30 \mathrm{~Hz}, & t \in[10,15) \mathrm{s}
\end{array} .\right.
$$

In Fig. 3 we show the result of this test. The controller providing the best behavior is selected quickly by the supervisor, thus ensuring a very good performance level. Further, thanks to the specific design technique, which guarantees the existence of a common Lyapunov function for the feedback loops, the transitions between the controllers are smooth.

\section{CONCLUSIONS}

This paper proposes an algorithm for the design of a set of controllers to be used within an adaptive switching control scheme with the aim of attenuating the effects of disturbances having uncertain (and possibly time-varying) characteristics. Specifically, the problem of ensuring stability under arbitrary switching is addressed, by accounting for this requirement directly within the design of the controller set by means of LMI constraints. The algorithm turns out to be well suited to dealing with the context of disturbances described by uncertain models, as well as to being solved by means of standard tools for convex optimization problems. The validity of the proposed method is underlined by means of simulation tests on an adaptive optics case study.

\section{REFERENCES}

[1] A. S. Morse, D. Q. Mayne, and G. C. Goodwin, "Applications of hysteresis switching in parameter adaptive control," IEEE Transactions on Automatic Control, vol. 37, pp. 1343-1354, 1992.

[2] K. S. Narendra and J. Balakrishnan, "Adaptive control using multiple models," IEEE Transactions on Automatic Control, vol. 42, pp. 171187, 1997.

[3] J. Hespanha, D. Liberzon, A. S. Morse, B. D. O. Anderson, T. S. Brinsmead, and F. De Bruyne, "Multiple model adaptive control, part 2: switching," International Journal of Robust and Nonlinear Control, vol. 11(5), pp. 479-496, 2001.

[4] J. P. Hespanha, D. Liberzon, and A. S. Morse, "Hysteresis-based switching algorithms for supervisory control of uncertain systems," Automatica, vol. 39, pp. 263-272, 2003.

[5] S. Baldi, G. Battistelli, E. Mosca, and P. Tesi, "Multi-model unfalsified adaptive switching supervisory control," Automatica, vol. 46, no. 2, pp. 249-259, 2010.

[6] G. Battistelli, J. P. Hespanha, and P. Tesi, "Supervisory control of switched nonlinear systems," International Journal of Adaptive Control and Signal Processing, vol. 26, no. 8, pp. 723-738, 2012.

[7] G. Battistelli, J. P. Hespanha, E. Mosca, and P. Tesi, "Model-free adaptive switching control of time-varying plants," IEEE Transactions on Automatic Control, vol. 58, no. 5, pp. 1208-1220, 2013.

[8] R. Wang, A. Paul, M. Stefanovic, and M. G. Safonov, "Cost detectability and stability of adaptive control systems," International Journal of Robust and Nonlinear Control, vol. 17 (5-6), pp. 549-561, 2007.

[9] H. Jin and M. G. Safonov, "Unfalsified adaptive control: Controller switching algorithms for nonmonotone cost functions," International Journal of Adaptive Control and Signal Processing, vol. 26 (8), pp. 692-704, 2012.

[10] G. Battistelli, E. Mosca, and P. Tesi, "Adaptive memory in multimodel switching control of uncertain plants," Automatica, vol. 50(3), pp. 874-882, 2014.

[11] G. Battistelli, D. Mari, D. Selvi, A. Tesi, and P. Tesi, "Adaptive disturbance attenuation via logic-based switching," Systems \& Control Letters, vol. 73, pp. 48-57, 2014.

[12] G. Battistelli, D. Mari, D. Selvi, A. Tesi, and P. Tesi, "A Hierarchical Approach to Adaptive Disturbance Attenuation Combining Switching and Tuning," Proceedings of the 53rd IEEE Conference on Decision and Control, Los Angeles, California, USA, pp. 6254-6259, 2014.

[13] J. C. Geromel and P. Colaneri, "Stability and stabilization of discrete time switched systems," International Journal of Control, vol. 79(7), pp. 719-728, 2006.

[14] D. Liberzon, Switching in Systems and Control. Birkhauser, 2003.

[15] D. Efimov, A. Loria, and E. Panteley, "Robust output stabilization: Improving performance via supervisory control," International Journal of Robust and Nonlinear Control, vol. 21(10), pp. 1219-1236, 2011.

[16] A. Karimi and G. Galdos, "Fixed-order $H_{\infty}$ controller design for nonparametric models by convex optimization," Automatica, vol. 46, no. 8, pp. 1388-1394, 2010.

[17] A. Karimi and Z. Emedi, " $H_{\infty}$ gain-scheduled controller design for rejection of time-varying narrow-band disturbances applied to a benchmark problem," European Journal of Control, vol. 19, pp. 279288, 2013.

[18] M. S. Sadabadi and A. Karimi, "An LMI formulation of fixed-order $H_{\infty}$ and $H_{2}$ controller design for discrete-time systems with polytopic uncertainty," Proceedings of the 52nd IEEE Conference on Decision and Control, Florence, Italy, pp. 2453-2458, 2013.

[19] P. S. C. Heuberger, P. M. J. Van Den Hof, and B. Wahlberg, Modelling and identification with rational orthogonal basis functions. Springer, 2004.

[20] G. Agapito, G. Battistelli, D. Mari, D. Selvi, A. Tesi, and P. Tesi, "Frequency based design of modal controllers for adaptive optics systems," Optics Express, vol. 20, no. 24, pp. 27 108-27 122, 2012.

[21] G. Agapito, F. Quiros-Pacheco, P. Tesi, A. Riccardi, and S. Esposito, "Observer-based control techniques for the LBT adaptive optics under telescope vibrations," European Journal of Control, vol. 17, no. 3, 2011. 